\title{
The interphase finite element
}

\section{Giuseppe Giambanco, Giuseppe Fileccia Scimemi \& Antonino Spada}

\section{Computational Mechanics}

Solids, Fluids, Structures, Fluid-

Structure Interactions, Biomechanics, Micromechanics, Multiscale Mechanics, Materials, Constitutive Modeling, Nonlinear Mechanics, Aerodynamics

ISSN 0178-7675

Comput Mech

DOI 10.1007/s00466-011-0664-8

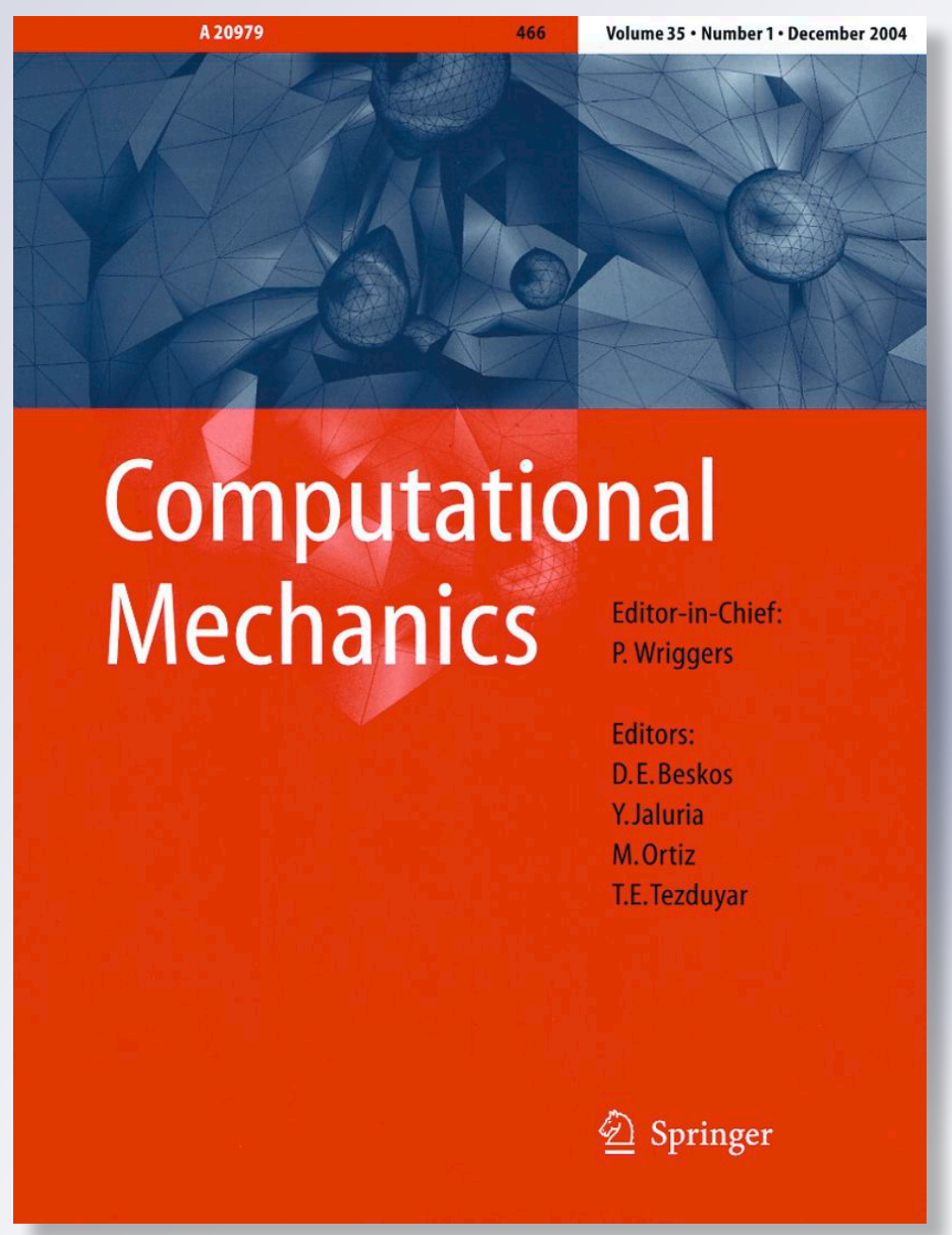

Springer 
Your article is protected by copyright and all rights are held exclusively by SpringerVerlag. This e-offprint is for personal use only and shall not be self-archived in electronic repositories. If you wish to self-archive your work, please use the accepted author's version for posting to your own website or your institution's repository. You may further deposit the accepted author's version on a funder's repository at a funder's request, provided it is not made publicly available until 12 months after publication. 


\title{
The interphase finite element
}

\author{
Giuseppe Giambanco • Giuseppe Fileccia Scimemi • \\ Antonino Spada
}

Received: 17 December 2010 / Accepted: 1 November 2011

(c) Springer-Verlag 2011

\begin{abstract}
Mesomodelling of structures made of heterogeneous materials requires the introduction of mechanical models which are able to simulate the interactions between the adherents. Among these devices is quite popular the zero thickness interface (ZTI) model where the contact tractions and the displacement discontinuities are the primary static and kinematic variables. In some cases the joint response depends also on the internal stresses and strains within the thin layer adjacent to the joint interfaces. The interphase model, taking into account these additional variables, represents a sort of enhanced ZTI. In this paper a general theoretical formulation of the interphase model is reported and an original finite element, suitable for two-dimensional applications, is presented. A simple numerical experiment in plane stress state condition shows the relevant capabilities of the interphase element and allows to investigate its numerical performance. Some defects related to the shear locking of the element are resolved making use of well known numerical strategies. Finally, further numerical application to masonry structures are developed.
\end{abstract}

Keywords Heterogeneous materials - Mesomodelling · Interphase · Finite element

\section{Introduction}

All those structures constituted by an heterogeneous material, such as masonry structures and composite laminates,

G. Giambanco $(\varangle) \cdot$ G. Fileccia Scimemi · A. Spada

Department of Civil, Environmental and Aerospace Engineering, University of Palermo, Viale delle Scienze, 90128 Palermo, Italy

e-mail: giuseppe.giambanco@unipa.it present a mechanical response strongly affected by the static and kinematic phenomena occurring at each constituent and at their joints. Therefore, some different length scales of interest may be identified and for each scale some specific deformation mechanisms need to be investigated.

First of all, the structural or macroscopic length scale is of the order of the typical dimensions of the structural element. The mechanical variables defined at this scale are related to some average material properties (homogeneous equivalent material) and consequently stresses and strains are to be considered as average stress and strain fields.

Next, the mesoscopic length scale is of the order of the typical dimension of the basic constituents of the heterogeneous material. The stress and strain at each constituent represent the variables at this scale and some effects, such as damage-induced anisotropy, which is observed at the macroscopic scale, is mainly governed by the damage growth taking place at the mesoscopic scale.

Finally, a lower length scale may be identified for each constituent and the relevance of this scale arises in micromechanics problems.

The overall macroscopic approach to the analysis of structures made up of heterogeneous material consists in formulating phenomenological constitutive laws expressed in terms of macroscopic stress and average strain for the equivalent homogeneous continuum [1-4]. Mathematical relations describe the behaviour of a number of phases and the macroscopic behaviour of the volume element is obtained by applying more or less complex averaging processes to these relations.

This way to operate may be not adequate to describe the non linear behaviour since it requires the introduction of strong simplifications. The fact is that non-linearities really develop in a discrete framework, mainly as debonding, sliding and other effects which occur locally between 
components. The equivalent average procedure could easily fail in the proper description of the material behaviour since it is not able to take into account the specific interaction between elements.

The mesoscopic approach overcomes these difficulties since the constituents are modelled individually and their interactions are regulated by apposite interface models [5-17]. This approach is versatile and allows to capture accurately the principal failure mechanisms.

The mesoscopic approach can be easily implemented in the framework of the finite element method (FEM) for the purpose of numerical modelling of heterogeneous structures. The material constituents are modelled making use of the classical 2D or 3D continuum elements while the joints are simulated through mechanical devices able to reproduce opening-closing, slide and dilatancy phenomena. These mechanical devices, generally called contact elements, are classified in the following categories:

- link elements between two opposite nodes [18] or a node and a segment [19] of the elements in contact;

- continuum finite elements of small but finite thickness sometimes referred as thin layer elements [20];

- zero thickness interface (ZTI) elements in which the displacements discontinuities between the top and bottom sides of the element represent the primary kinematic variables [21].

The ZTI elements in recent years have found several applications in civil and industrial engineering. The mechanical response of mortar joints in masonry material and of the steel bar/hardened concrete bond surface in reinforced concrete members are simulated making use of ZTI elements. The same elements are used to study laminated composites to describe delamination phenomena.

A wide literature is devoted to the development of advanced interface constitutive models in order to describe the damage evolution, the onset of irreversible strains and the related coupled effects. The constitutive laws are expressed in terms of contact tractions and displacement discontinuities which are considered as generalized joint strains. Along the loading path, most of the proposed interface laws describe a linear elastic response until a failure criterion is satisfied. The evolutive constitutive equations describing the post peak softening response are often formulated in rigorous manner and incorporate some concepts developed in damage mechanics [10-13], in the theory of plasticity for non-standard materials $[9,32]$ and in fracture mechanics [14].

A number of papers deal with the numerical performance of the ZTI elements. The earliest FEM application which introduce ZTI elements is attributed to Goodman [21] that in the late sixties handled the two-dimensional mechanical response of rock masses. Later, the isoparametric formula- tion for interface elements was proposed by Beer [22] to model contact surfaces or thin layer joints in three-dimensional structures. Extension to finite deformations was developed by Ortiz and Pandolfi [23].

This kind of interface elements, as reported by Schellekens and de Borst [24], show undesired spurious oscillations of the stress field, in particular when the interface stiffness is high respect to that of surrounding elements. The authors relate this drawback to the applied numerical integration scheme of the stiffness matrix and propose a numerical strategy to overcome the stress field oscillation. The same deficiency has been noted by Kaliakin and $\mathrm{Li}[25]$ and this is reconducted to a kinematic inconsistency of the element. In the cited paper an improved four-node element is proposed which is the result of the assembly of two aligned four noded interface elements by using the static condensation technique.

In many cases the joint response depends also on internal stresses and strains within the contact layer adjacent to the joint interfaces. A typical example is the uniaxial compression of masonry material. With reference to the Fig. 1, the masonry specimen is constituted by two blocks interfaced by a mortar joint. The overall mechanical response depends on the ratio between the Young modulus of the block and of the mortar. First, we distinguish the case of a mortar stiffer than the block material (a). In this case the joint provides a sort of confinement action on the two blocks resulting in the onset of tangential stresses at the physical joint/block interfaces. Considering the tangential contact tractions as an external load for the mortar thin layer, the equilibrium gives that the transverse section of the joint is subjected to a tensile normal stress. On the other hand, a block material stiffer than the mortar (b) produces the opposite effect: the mortar squeezing and the confinement action provided by the blocks lead to compressive normal stresses in the transverse section. Furthermore, the tangential contact tractions produce a sort of bending effect for the block which is the cause of the fracture experimentally observed departing from the inner horizontal surface of the unit.

The ZTI concept applied to the reported example is not able to capture the illustrated response of the masonry specimen and, in terms of contact tractions, provides compression in the normal direction and zero tangential tractions.

Therefore, the usual assumption used in ZTI that the response is governed by contact stresses components, may require an enhancement by introducing the effect of the internal stresses into the analysis.

In this paper, we shall study the mesomodelling of heterogeneous materials making use of the interphase model proposed by Giambanco and Mroz [26]. The principal aim is the formulation of a reliable contact finite element based on the kinematics of the interphase model and representing a thin layer joint separated by two interfaces from the adherents. The introduced novelties are that the internal stresses 


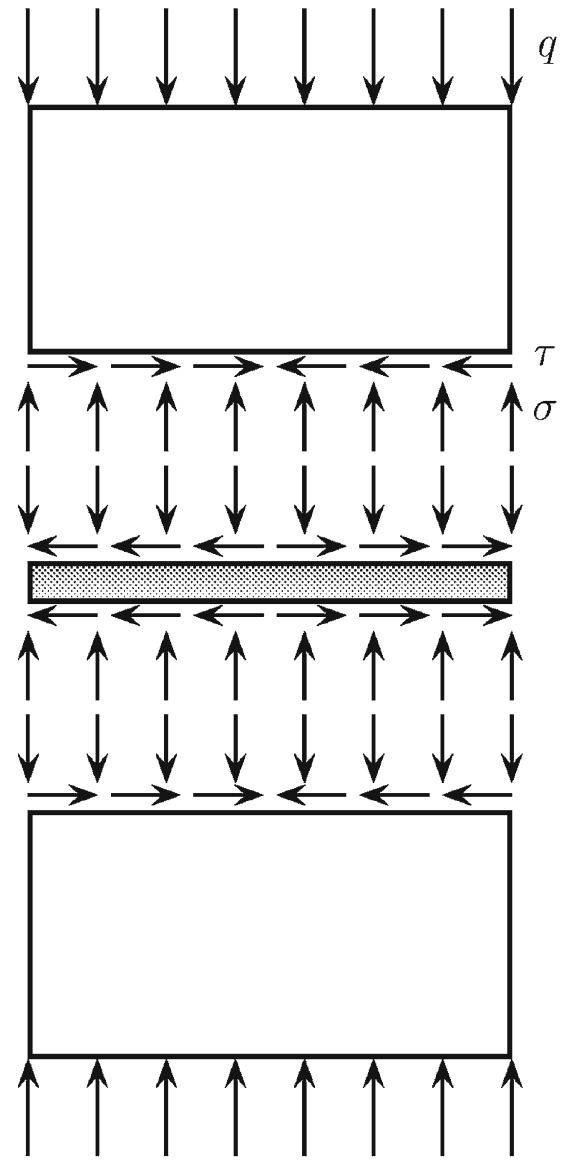

(a) $E_{m}>E_{b}$

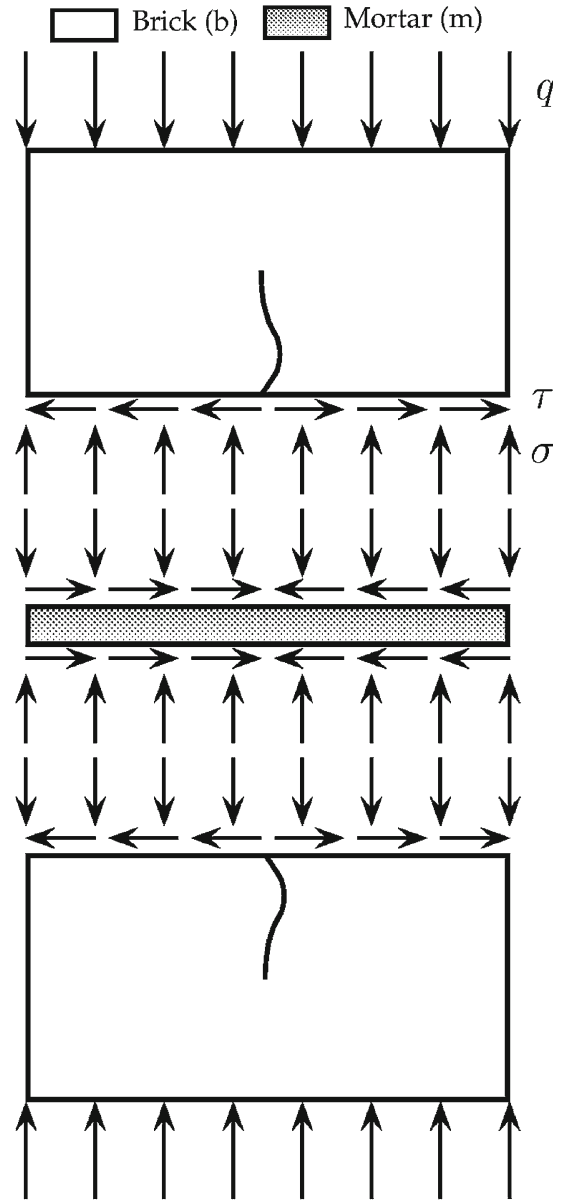

(b) $E_{b}>E_{m}$

Fig. 1 Uniaxial compression test on masonry specimen

are incorporated and the contact stiffness is evaluated from the elastic properties of the joint material.

The paper is structured as follows: in the Sect. 2, for the sake of completeness, the interphase model is illustrated in a general manner, as in the original formulation, providing the kinematics and equilibrium equations. Afterwards, the mechanical problem is specialized for the case of twodimensional analysis under plane stress condition and the new linear elastic interphase element is formulated in Sect. 3. The finite element is implemented in a scientific oriented finite element code and the numerical performances are investigated in detail in Sect. 4. In particular, a sort of patch test is proposed to study the convergence properties of the element and, since some drawbacks of the element are verified, well known numerical strategies used to solve locking effects in thin 2D and 3D continuum elements are applied. Numerical elastic applications are developed in Sect. 5 for masonry structures subjected to more complex load combinations. In Sect. 6 the conclusions and the future developments are reported.

\section{General assumptions}

Let us consider, in the Euclidean space $\mathbb{R}^{3}$ referred to the orthonormal frame $\left(\mathrm{O}, \mathbf{i}_{1}, \mathbf{i}_{2}, \mathbf{i}_{3}\right)$, a structure formed by two adherents $\Omega^{+}, \Omega^{-}$connected by a third material or joint $\Omega^{j}$, see Fig. 2.

We assume that the thickness $h$ of the joint is small if compared with the characteristic dimensions of the bonded assembly.

The two adherents have Lipschitz boundaries $\partial \Omega^{ \pm}$and the joint $\Omega^{j}$ interacts with the two adherents through the two physical interfaces $\Sigma^{+}$and $\Sigma^{-}$defined as follows:

$\Sigma^{+}=\partial \Omega^{+} \cap \Omega^{j}, \quad \Sigma^{-}=\partial \Omega^{-} \cap \Omega^{j}$.

The remaining parts of the adherents boundary are divided in two parts: the part $\Gamma_{u}^{ \pm}$where kinematic conditions are applied and the part $\Gamma_{t}^{ \pm}$where tractions are specified.

The static and kinematic quantities of the joint are referred to the orthonormal frame $\left(\mathrm{O}, \mathbf{e}_{1}, \mathbf{e}_{2}, \mathbf{e}_{3}\right)$ with $\mathbf{e}_{3}$ oriented 


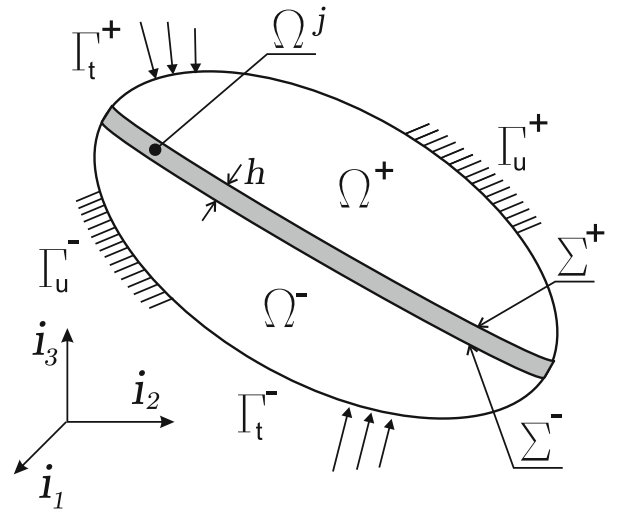

Fig. 2 The mechanical problem of a third body interposed between adherents

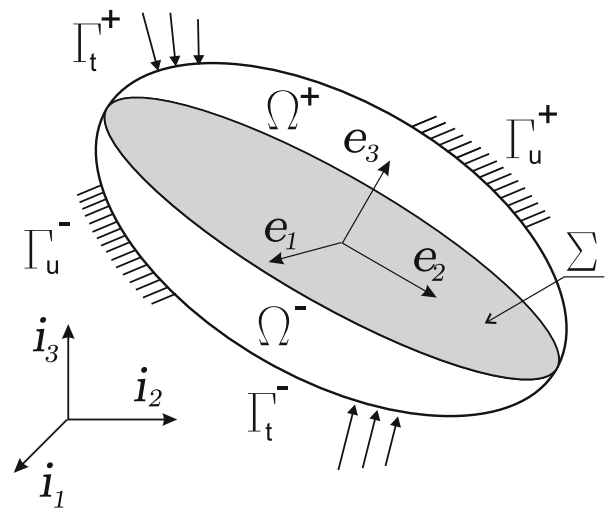

Fig. 3 The schematic representation of the mechanical problem using the interphase model

along the normal to the middle surface $\Sigma$ and directed into the adherent $\Omega^{+}$, Fig. 3. The joint interacts with the two adherents through the following traction components:

$\mathbf{t}^{+}=t_{1}^{+} \mathbf{e}_{1}+t_{2}^{+} \mathbf{e}_{2}+t_{3}^{+} \mathbf{e}_{3}, \quad \mathbf{t}^{-}=t_{1}^{-} \mathbf{e}_{1}+t_{2}^{-} \mathbf{e}_{2}+t_{3}^{-} \mathbf{e}_{3}$

which can be considered as external surface loads for the joint.

The joint can be regarded as an interphase model where the fibres directed along the normal to the middle surface are maintained rectilinear along the deformation process. In view of this hypothesis the interphase displacement field $\mathbf{u}$ can be easily obtained from the displacements $\mathbf{u}^{+}$and $\mathbf{u}^{-}$of the interfaces $\Sigma^{+}$and $\Sigma^{-}$, thus

$$
\begin{aligned}
\mathbf{u}\left(x_{1}, x_{2}, x_{3}\right)= & \left(\frac{1}{2}+\frac{x_{3}}{h}\right) \mathbf{u}^{+}\left(x_{1}, x_{2}\right) \\
& +\left(\frac{1}{2}-\frac{x_{3}}{h}\right) \mathbf{u}^{-}\left(x_{1}, x_{2}\right)
\end{aligned}
$$

with $\left(x_{1}, x_{2}, x_{3}\right)$ a Cartesian coordinate system associated to the interphase orthonormal frame.

Since the thickness of the joint is generally small with respect to other two dimensions, we can assume a representative strain state $\boldsymbol{\varepsilon}$ constant along $\mathbf{e}_{3}$ direction, thus $\boldsymbol{\varepsilon}\left(x_{1}, x_{2}\right)=\frac{1}{h} \int_{-h / 2}^{h / 2} \nabla^{s} \mathbf{u}\left(x_{1}, x_{2}, x_{3}\right) d x_{3}$

and substituting the expression (3) we have

$\boldsymbol{\varepsilon}\left(x_{1}, x_{2}\right)=\frac{1}{2 h}\left([\mathbf{u}] \otimes \mathbf{I}_{3}+[\mathbf{u}] \otimes \mathbf{I}_{3}\right)+\frac{1}{2} \nabla^{s}\left(\mathbf{u}^{+}+\mathbf{u}^{-}\right)$,

where $[\mathbf{u}]=\mathbf{u}^{+}-\mathbf{u}^{-}, \mathbf{I}_{3}=\left\{\delta_{i 3}\right\}$ and $\nabla^{s}$ is the symmetric gradient operator.

Let us note that in the interphase model the joint curvatures generated by the displacement field (3) and the related flexural effects are neglected.

In order to derive the equilibrium equations of the interphase model, let us assign the virtual displacements $\delta \mathbf{u}^{+}$and $\delta \mathbf{u}^{-}$at the interfaces $\Sigma^{+}$and $\Sigma^{-}$. The principle of virtual displacements $(P V D)$ asserts that the external work, produced by the contact tractions, equals the internal work developed in the thin joint. According to the hypothesis of a constant strain state along the thickness direction, the conjugate stress state is considered uniform along the same direction and the $P V D$ assume the following form:

$\int_{\Sigma^{+}} \delta \mathbf{u}^{+} \cdot \mathbf{t}^{+} d \Sigma+\int_{\Sigma^{+}} \delta \mathbf{u}^{-} \cdot \mathbf{t}^{-} d \Sigma=h \int_{\Sigma} \delta \boldsymbol{\varepsilon}: \sigma d \Sigma$.

Taking into account the kinematic relations (5) the virtual work equation (6) becomes

$$
\begin{aligned}
& \int_{\Sigma^{+}} \delta \mathbf{u}^{+} \cdot \mathbf{t}^{+} d \Sigma+\int_{\Sigma^{-}} \delta \mathbf{u}^{-} \cdot \mathbf{t}^{-} d \Sigma \\
& =\frac{1}{2} \int_{\Sigma}\left(\delta[\mathbf{u}] \otimes \mathbf{I}_{\mathbf{3}}+\mathbf{I}_{\mathbf{3}} \otimes \delta[\mathbf{u}]\right): \sigma d \Sigma \\
& \quad+\frac{h}{2} \int_{\Sigma}\left[\nabla^{s}\left(\delta \mathbf{u}^{+}+\delta \mathbf{u}^{-}\right)\right]: \sigma d \Sigma .
\end{aligned}
$$

Let us apply the divergence theorem to the second term of the right-hand side in the Eq. 7, we have

$$
\begin{aligned}
& \int_{\Sigma}\left[\nabla^{s}\left(\delta \mathbf{u}^{+}+\delta \mathbf{u}^{-}\right)\right]: \sigma d \Sigma=\int_{\Gamma} \mathbf{m} \cdot \boldsymbol{\sigma} \cdot\left(\delta \mathbf{u}^{+}+\delta \mathbf{u}^{-}\right) d \Gamma \\
& -\int_{\Sigma}\left(\delta \mathbf{u}^{+}+\delta \mathbf{u}^{-}\right) \cdot \operatorname{div} \boldsymbol{\sigma} d \Sigma
\end{aligned}
$$

where $\Gamma$ represents the perimeter of the joint middle plane $\Sigma$ and $\mathbf{m}$ is the normal specifying lateral surface tractions. Substituting equation (8) in the equality (7) and assuming that $\Sigma=\Sigma^{+}=\Sigma^{-}$, we derive the $P V D$ particularized for the interphase model, namely: 


$$
\begin{aligned}
& \int_{\Sigma} \delta \mathbf{u}^{+} \cdot\left(\mathbf{t}^{+}-\boldsymbol{\sigma} \cdot \mathbf{I}_{3}+\frac{h}{2} \operatorname{div} \boldsymbol{\sigma}\right) d \Sigma \\
& +\int_{\Sigma} \delta \mathbf{u}^{-} \cdot\left(\mathbf{t}^{-}+\boldsymbol{\sigma} \cdot \mathbf{I}_{3}+\frac{h}{2} \operatorname{div} \boldsymbol{\sigma}\right) d \Sigma \\
= & \frac{h}{2} \int_{\Gamma} \mathbf{m} \cdot \boldsymbol{\sigma} \cdot\left(\delta \mathbf{u}^{+}+\delta \mathbf{u}^{-}\right) d \Gamma .
\end{aligned}
$$

Since the previous equality holds for all virtual displacements fields, it generates the local equilibrium relations of the interphase model:

$$
\mathbf{t}^{+}-\boldsymbol{\sigma} \cdot \mathbf{I}_{3}+\frac{h}{2} \operatorname{div} \boldsymbol{\sigma}=0, \quad \mathbf{t}^{-}+\sigma \cdot \mathbf{I}_{3}+\frac{h}{2} \operatorname{div} \sigma \quad \text { on } \Sigma
$$

$\mathbf{m} \cdot \boldsymbol{\sigma}=0$ on $\Gamma$.

\section{The isoparametric interphase element}

For the purpose of mesoscopic numerical analysis of structures made of an heterogeneous material, the interphase model presented in the previous section has been implemented in the framework of FEM. The case developed here regards two-dimensional structures referred to the $\left(x_{1}, x_{3}\right)$ plane with linear elastic response of the adherents and of the joint, in the hypothesis of plane stress state condition.

The extension to the plane strain or to the axial symmetric cases is straightforward. Applications to three-dimensional structures will be the scope of a future work.

Making use of matrix algebra notation, the elastic constitutive relation of the interphase material is:

$\sigma=\mathbf{E} \varepsilon$

where the stress and strain vectors are

$$
\begin{aligned}
& \boldsymbol{\sigma}=\left[\begin{array}{lll}
\sigma_{x 1} & \sigma_{x 3} & \tau_{x 1 x 3}
\end{array}\right]^{\mathrm{T}}, \\
& \boldsymbol{\varepsilon}=\left[\begin{array}{lll}
\varepsilon_{x 1} & \varepsilon_{x 3} & \gamma_{x 1} x_{3}
\end{array}\right]^{\mathrm{T}}
\end{aligned}
$$

and $\mathbf{E}$ represents the elasticity matrix. The interphase material is assumed isotropic, therefore the elasticity matrix and the out of plane normal strain assume the following form:

$$
\begin{aligned}
& \mathbf{E}=\frac{E}{1-v^{2}}\left[\begin{array}{ccc}
1 & v & 0 \\
v & 1 & 0 \\
0 & 0 & \frac{1-v}{2}
\end{array}\right], \\
& \varepsilon_{x 2}=-\frac{v}{E}\left(\sigma_{x 1}+\sigma_{x 3}\right),
\end{aligned}
$$

with $E$ and $v$ the elasticity modulus and the Poisson's ratio of the joint material, respectively.

The interphase element developed has four nodes and is compatible with the classical 2D solid elements with linear

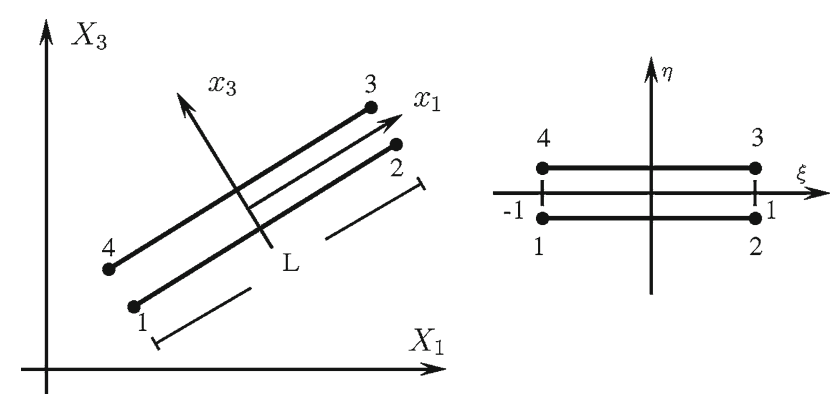

Fig. 4 The four noded interphase element

shape functions. The isoparametric formulation of the element considers the local coordinate system $(\xi, \eta)$ located in the midpoint of the element, see Fig. 4. Each node has two displacement degrees of freedom, therefore the kinematic variables of the element are represented by the nodal displacement vector $\mathbf{d}$

$\mathbf{d}=\left[\begin{array}{l}\mathbf{d}^{-} \\ \mathbf{d}^{+}\end{array}\right]$

where

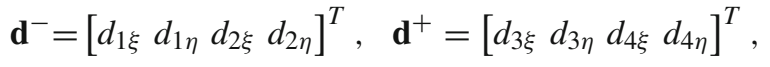

are the displacement of the bottom $(-)$ and the top $(+)$ nodes of the element. Making use of the following linear shape functions

$N_{1}=\frac{1}{2}(1-\xi), \quad N_{2}=\frac{1}{2}(1+\xi)$,

the continuous displacement fields of the upper and lower side of the interphase can be expressed in terms of the nodal displacements, thus

$\mathbf{u}^{+}=\mathbf{N}^{+} \mathbf{d}^{+}, \quad \mathbf{u}^{-}=\mathbf{N}^{-} \mathbf{d}^{-}$,

where

$\mathbf{N}^{+}=\left[\begin{array}{cccc}N_{2} & 0 & N_{1} & 0 \\ 0 & N_{2} & 0 & N_{1}\end{array}\right], \quad \mathbf{N}^{-}=\left[\begin{array}{cccc}N_{1} & 0 & N_{2} & 0 \\ 0 & N_{1} & 0 & N_{2}\end{array}\right]$.

The strain state of the isoparametric element is obtained from Eq. 5, particularized for the plane stress case, and from the interpolated displacement fields (20):

$\varepsilon=\mathbf{B d}$

where the kinematic compatibility matrix $\mathbf{B}$ can be additively decomposed in two parts:

$\mathbf{B}=\mathbf{B}_{t}+\mathbf{B}_{p h}$.

The first one relates the discontinuous displacement field to the nodal displacements and is used in the classical ZTI element. The latter is employed in the interphase element to take into account the internal strains. The explicit form of the two matrices is: 
$\mathbf{B}_{t}=\frac{1}{h}\left[\begin{array}{cccccccc}0 & 0 & 0 & 0 & 0 & 0 & 0 & 0 \\ 0 & -N_{1} & 0 & -N_{2} & 0 & N_{2} & 0 & N_{1} \\ -N_{1} & 0 & -N_{2} & 0 & N_{2} & 0 & N_{1} & 0\end{array}\right]$,

$\mathbf{B}_{p h}=\frac{1}{L}\left[\begin{array}{cccccccc}N_{1}^{\prime} & 0 & N_{2}^{\prime} & 0 & N_{2}^{\prime} & 0 & N_{1}^{\prime} & 0 \\ 0 & 0 & 0 & 0 & 0 & 0 & 0 & 0 \\ 0 & N_{1}^{\prime} & 0 & N_{2}^{\prime} & 0 & N_{2}^{\prime} & 0 & N_{1}^{\prime}\end{array}\right]$,

where the prime symbol designates the first derivative of the function respect to the spatial variable $\xi$.

Let us note that in order to simplify the equations governing the finite element, the interphase local co-ordinate system coincides with the global co-ordinate system and no transformation is necessary. For an arbitrary oriented interphase element, the matrix $\mathbf{B}$ has to be transformed.

Considering the interphase element of thickness $h$, width $b$ and length $L$, the total potential energy of the element equals:

$$
\begin{aligned}
\Pi(\boldsymbol{\varepsilon}, \mathbf{u})= & \frac{b h L}{4} \int_{-1}^{1} \boldsymbol{\varepsilon}^{T} \mathbf{E} \boldsymbol{\varepsilon} d \xi \\
& -\frac{b \cdot L}{2} \int_{-1}^{1}\left(\mathbf{t}^{+^{T}} \mathbf{u}^{+}+\mathbf{t}^{-T} \mathbf{u}^{-}\right) d \xi
\end{aligned}
$$

which after invoking equations (20) and (22) results in

$$
\Pi(\mathbf{d})=\frac{b \cdot h \cdot L}{4} \int_{-1}^{1} \mathbf{d}^{T} \mathbf{B}^{T} \mathbf{E} \mathbf{B} \mathbf{d} d \xi-\frac{b \cdot L}{2} \int_{-1}^{1} \mathbf{t}^{T} \mathbf{N} \mathbf{d} d \xi,
$$

with the following positions

$\mathbf{t}=\left[\begin{array}{l}\mathbf{t}^{-} \\ \mathbf{t}^{+}\end{array}\right], \quad \mathbf{N}=\left[\begin{array}{cc}\mathbf{N}^{-} & \mathbf{0} \\ \mathbf{0} & \mathbf{N}^{+}\end{array}\right]$

Using the standard procedure of minimizing the total potential energy with respect to the nodal displacements, the interphase element equilibrium equations are obtained, thus

$\mathbf{f}=\mathbf{K} \mathbf{d}$

where the stiffness matrix $\mathbf{K}$ and the nodal force vector $\mathbf{f}$ are expressed as

$$
\begin{aligned}
\mathbf{K} & =\frac{b \cdot h \cdot L}{2} \int_{-1}^{1} \mathbf{B}^{T} \mathbf{E} \mathbf{B} \mathrm{d} \xi \\
\mathbf{f} & =\frac{b \cdot L}{2} \int_{-1}^{1} \mathbf{N}^{T} \mathbf{t} d \xi
\end{aligned}
$$

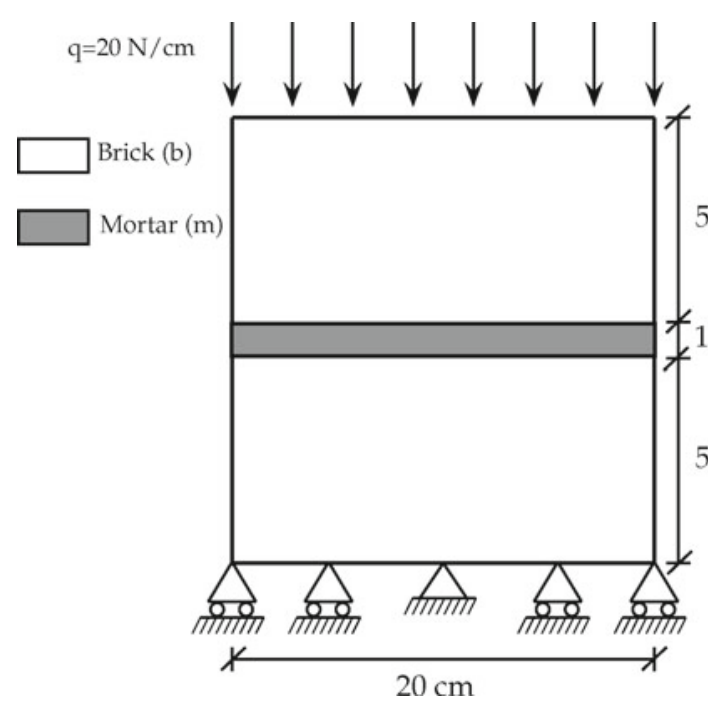

Fig. 5 The mechanical model of the uniaxial compression of masonry specimen

\section{Element numerical performance}

The numerical performance of the interphase element has been assessed making use of a sort of patch test regarding the simple linear elastic response of two masonry blocks joined by a mortar thin layer subjected to uniaxial compression. The geometry, kinematical constraints and load condition are illustrated in Fig. 5. Assuming the plane stress state, the model depth is posed equal to $1 \mathrm{~cm}$.

In the first set of numerical calculations the interphase stiffness matrix has been integrated by using the conventional Gauss quadrature scheme. The kinematic compatibility matrix is decomposed in a constant part and in a linear one:

$\mathbf{B}=\mathbf{B}_{0}+\mathbf{B}_{1} \xi$

the stiffness matrix (30) after integration takes the following form:

$$
\begin{aligned}
\mathbf{K} & =b \cdot h \cdot \frac{L}{2} \int_{-1}^{1}\left(\mathbf{B}_{0}+\mathbf{B}_{1} \xi\right)^{T} \mathbf{E}\left(\mathbf{B}_{0}+\mathbf{B}_{1} \xi\right) \mathrm{d} \xi \\
& =b \cdot h \cdot L\left(\mathbf{B}_{0}^{T} \mathbf{E} \mathbf{B}_{0}+\frac{1}{3} \mathbf{B}_{1}^{T} \mathbf{E} \mathbf{B}_{1}\right)
\end{aligned}
$$

The numerical tests performed regard two cases: in the first case the material of the block has the elastic modulus $E_{b}$ greater than the elastic modulus of the mortar $E_{m}$. In particular $E_{b}=10 E_{m}$. The second case is the opposite one and $E_{m}=30 E_{b}$. In order to test the numerical convergence of the model, the number of the interphase elements used varies from 10 to 80 . 
The numerical results for the first case are illustrated in Fig. 6 in terms of internal stress $\sigma_{x 1}$ and normal and tangential tractions $\sigma_{x 3}, \tau_{x 1 \times 3}$. The first goal attained is that, respect to the case of application of ZTI elements, the normal internal stress and the tangential contact traction appear. Further-
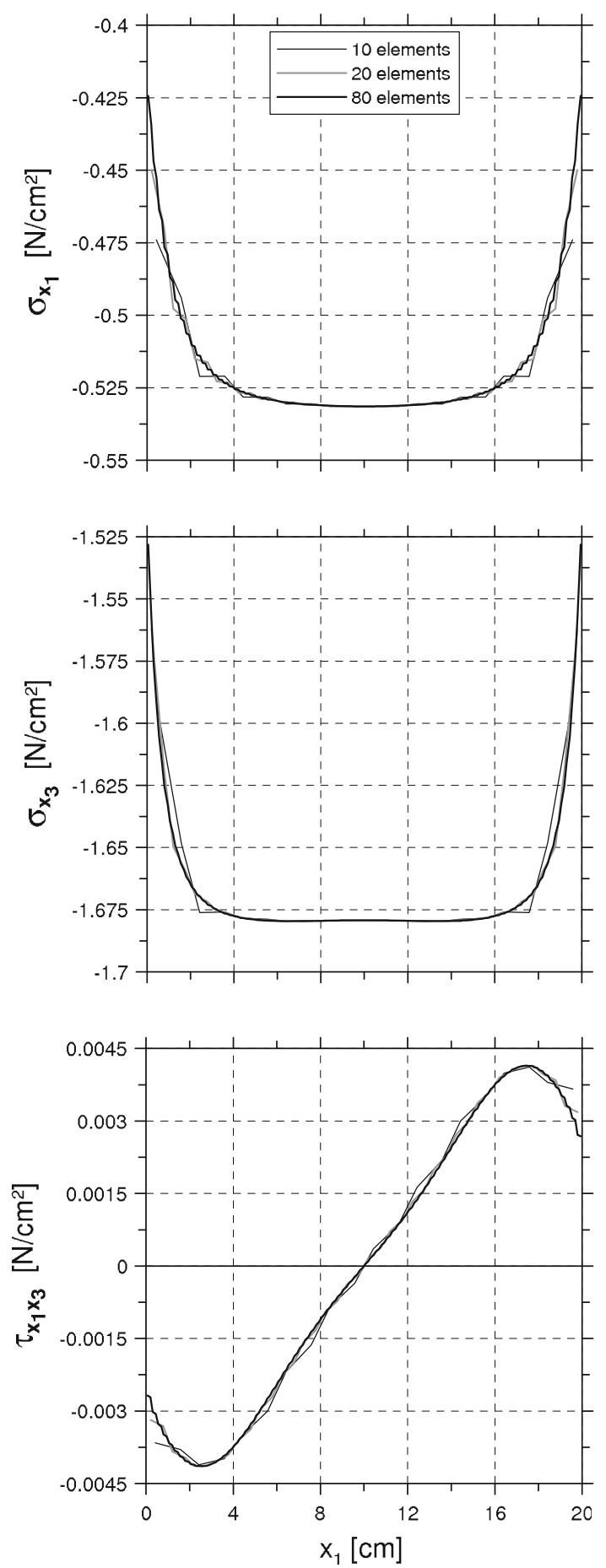

Fig. 6 Case $E_{b}=10 E_{m}$, standard Gauss quadrature, internal stress and contact tractions more, the progressive increment of the number of elements produces a more refined stress profile.

In the second case good performances of the element can be noticed for the internal stress and for the normal traction, Fig. 7. According to the theoretical treatment of the problem the mortar joint is subjected to a tensile normal stress in the cross section while before it was under compression. Unfortunately, bad results are obtained for the tangential traction showing an unacceptable oscillation of the profile which does not disappear increasing the number of elements used.

As mentioned in the introduction, similar spurious oscillations of the stress field have been observed by different authors in the ZTI elements when the joint is characterized by high values of the stiffness moduli and the sampling points of the element lie inside the joint, as in the case of the Gauss quadrature integration scheme. The bad element performance is connected to the fact that the element constraint matrix is coupled because of the interaction of the contributions from different shape functions. The study of the eigenvalues and eigenvectors of the matrix reveals that a sort of kinematic inconsistency exists in some of the deformation modes of the element [24].

The remedy usually adopted is to apply the nodal or Lobatto quadrature giving the decoupled constraint matrix, which become diagonal, since the contributions from the different shape functions do not interact. It is to observe that this way to solve the problem leads to a sort of degeneration of the ZTI element to discrete springs located at the nodes of the element.

Oscillation of the stress profiles are equally found in thinlayer elements. In thin 2D and 3D solid elements, but also in shear deformable beam and plate-shell elements, the spurious solutions in terms of stresses is the effect of the so called shear locking of the element which practically consists in an overestimation of the shear stiffness as soon as the aspect ratio of the element tends to zero. In order to avoid shear locking effects two strategies can be found in the literature:

- the Reduced or Selective Integration (RI-RSI) which provides "the necessary singularity of the constraint part of the stiffness matrix which avoids locking" $[27,28]$;

- the Enhanced Assumed Strain (EAS) method [29] where the strain field is enlarged and the element shows extra deformation modes.

The oscillation of the shear contact traction showed by the interphase element is equally imputable to shear locking since the element has a poor aspect ratio by definition. Therefore, the mentioned strategies are applied in the following to avoid the observed bad performances. 

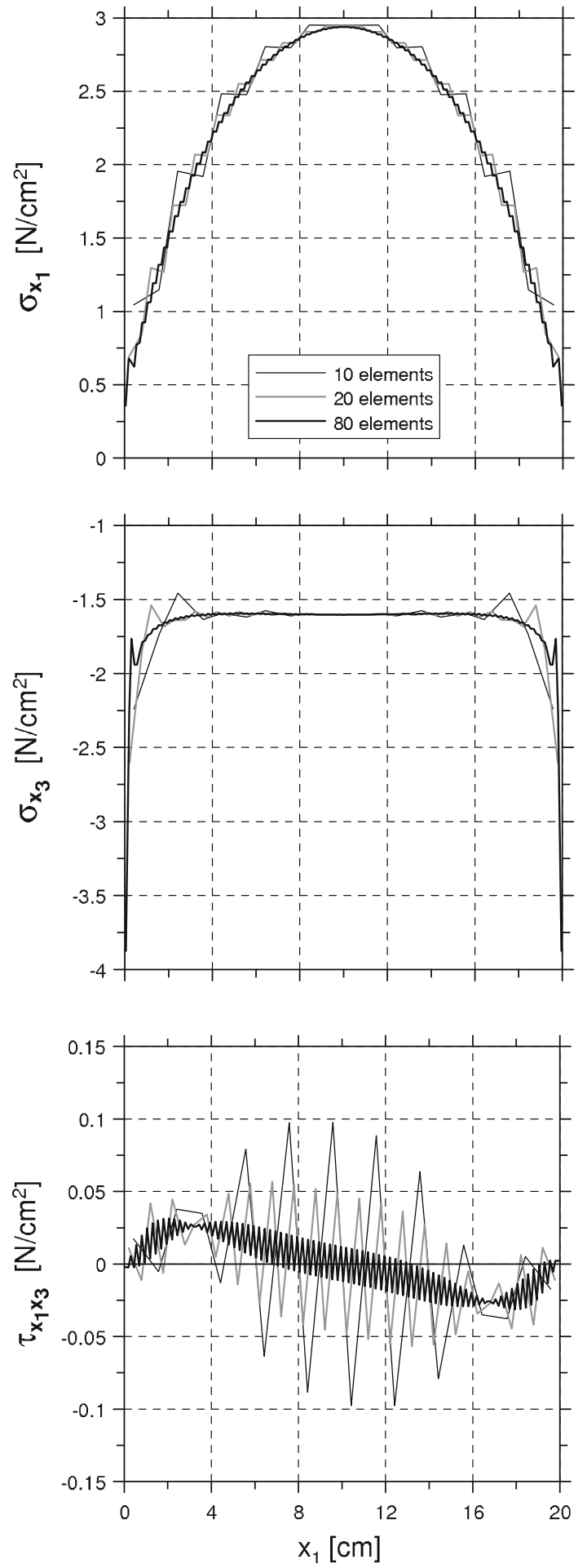

Fig. 7 Case $E_{m}=30 E_{b}$, standard Gauss quadrature, internal stress and contact tractions

\subsection{Reduced and reduced selective integration}

The reduced integration (RI) technique has been applied first, making use of a single Gauss point located in the middle of the element $(\xi=0)$. Therefore the explicit expression of stiffness matrix is:

$$
\begin{aligned}
\mathbf{K} & =b \cdot h \cdot \frac{L}{2} \int_{-1}^{1}\left(\mathbf{B}_{0}+\mathbf{B}_{1} \xi\right)^{T} \mathbf{E}\left(\mathbf{B}_{0}+\mathbf{B}_{1} \xi\right) \mathrm{d} \xi \\
& =b \cdot h \cdot L \mathbf{B}_{0}^{T} \mathbf{E} \mathbf{B}_{0} .
\end{aligned}
$$

The stiffness matrix obtained from the reduced integration is only a portion of the fully integrated one and, in particular, the portion containing the constant term of the compatibility matrix. The numerical results in terms of stresses making use of the reduced form of the stiffness matrix are illustrated in Fig. 8. The profile of the shear traction is now acceptable but the spurious oscillation is transferred to the normal contact stress.

The reason for this result is that the fully reduced integration introduces spurious modes which affect the response of the element with reference to the normal contact traction. This effect is well known [30] and can be demonstrated making use of the expression of the total potential energy rewritten taking into account the decomposition (32) of the B matrix:

$\Pi(\mathbf{d})=\Pi_{0}(\mathbf{d})+\Pi_{1 \varepsilon}(\mathbf{d})+\Pi_{1 \gamma}(\mathbf{d})$,

where $\Pi_{0}$ is the portion of the total potential energy whose density is constant in the element and $\Pi_{1 \varepsilon}, \Pi_{1 \gamma}$ are those parts whose density is variable. In particular, $\Pi_{1 \varepsilon}$ and $\Pi_{1 \gamma}$ are related to the extensional and shear strains respectively. The application of the RI strategy results in the following constraints for the element:

$\Pi_{1 \varepsilon}(\mathbf{d})=\Pi_{1 \gamma}(\mathbf{d})=0 ;$

all those element trial functions which satisfy equations (36) are referred as spurious modes.

The SRI technique was introduced as a way to avoid spurious modes. Essentially the selective reduced integration consists in using two different integration formulae: one for the shear part of the energy and a different one for the remaining part. Taking advantage of the obtained numerical results with the full and reduced integration, it is reasonable to apply the SRI technique in order to obtain:

$\Pi_{1 \varepsilon}(\mathbf{d}) \neq 0, \quad \Pi_{1 \gamma}(\mathbf{d})=0$.

The element stiffness matrix is further decomposed in the following form:

$$
\begin{aligned}
& \mathbf{K}=b \cdot h \cdot L \\
& \left(\mathbf{B}_{0}^{T} \mathbf{E} \mathbf{B}_{0}+\frac{G}{2} \int_{-1}^{1} \mathbf{b}_{3}^{T} \mathbf{b}_{3} \xi^{2} \mathrm{~d} \xi+\frac{E}{2\left(1-v^{2}\right)} \int_{-1}^{1} \mathbf{b}_{2}^{T} \mathbf{b}_{2} \xi^{2} \mathrm{~d} \xi\right),
\end{aligned}
$$



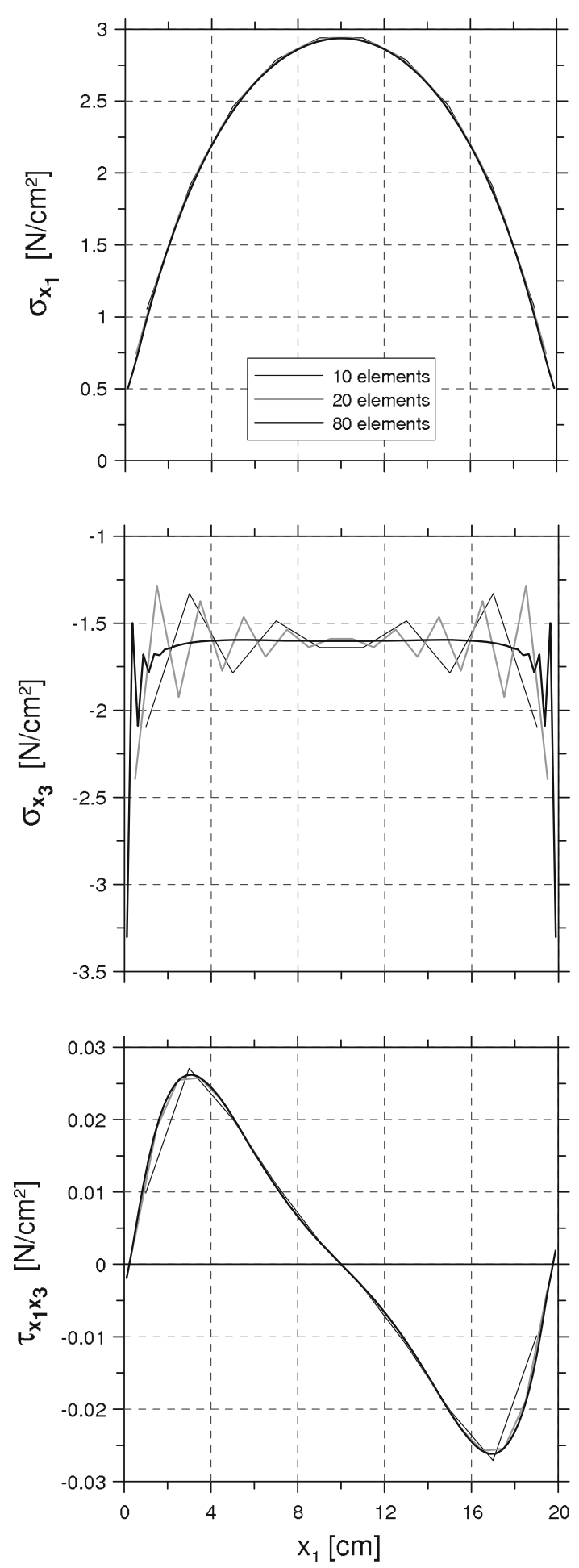

Fig. 8 Case $E_{m}=30 E_{b}$, reduced integration, internal stress and contact tractions

where $G$ is the tangential elastic modulus and the following partition of the $\mathbf{B}_{\mathbf{1}}$ matrix has been used:

$\mathbf{B}_{1}=\left[\begin{array}{c}\mathbf{0} \\ \mathbf{b}_{2} \\ \mathbf{b}_{3}\end{array}\right]$.
In order to satisfy conditions (37), the first integral of the right hand side of equation (38) is evaluated by a single Gauss point while the second is fully integrated. The final expression of the stiffness matrix is

$\mathbf{K}=b \cdot h \cdot L\left(\mathbf{B}_{0}^{T} \mathbf{E} \mathbf{B}_{0}+\frac{E}{3\left(1-v^{2}\right)} \mathbf{b}_{2}^{T} \mathbf{b}_{2}\right)$.

The SRI implemented in the interphase element routine provides the results reported in Fig. 9 and in both stress profiles, of the normal and tangential tractions, the oscillations disappear.

It can be concluded that the SRI strategy, applied as above illustrated, is effective for the improvement of the element numerical performance.

\subsection{Enhanced assumed strain}

The EAS strategy has been applied in the standard form presented in the work of Simo and Rifai [29]. The strain field is enlarged and the element shows extra deformation modes:

$\hat{\varepsilon}=\varepsilon+\varepsilon_{\alpha}$

where $\hat{\boldsymbol{\varepsilon}}$ is the enlarged strain vector and $\boldsymbol{\varepsilon}_{\alpha}$ is the enhanced part. The enhanced strains are derived by the interpolation of the additional kinematical parameters $\boldsymbol{\alpha}$ through the straindisplacement matrix $\mathbf{B}_{\alpha}$. Thus, from Eqs. 41 and 22 the enlarged strain vector equals:

$\hat{\boldsymbol{\varepsilon}}=\mathbf{B} \mathbf{d}+\mathbf{B}_{\alpha} \boldsymbol{\alpha}$

In linear elasticity, the element equilibrium equations can be written as follows:

$$
\left[\begin{array}{ll}
\mathbf{K}_{d d} & \mathbf{K}_{d \alpha} \\
\mathbf{K}_{\alpha d} & \mathbf{K}_{\alpha \alpha}
\end{array}\right]\left[\begin{array}{l}
\mathbf{d} \\
\boldsymbol{\alpha}
\end{array}\right]=\left[\begin{array}{l}
\mathbf{f} \\
\mathbf{0}
\end{array}\right]
$$

where the stiffness matrix is constituted by four blocks having the following expressions

$$
\begin{aligned}
& \mathbf{K}_{d d}=\frac{b \cdot h \cdot L}{2} \int_{-1}^{1} \mathbf{B}^{T} \mathbf{E} \mathbf{B} \mathrm{d} \xi ; \\
& \mathbf{K}_{d \alpha}=\frac{b \cdot h \cdot L}{2} \int_{-1}^{1} \mathbf{B}^{T} \mathbf{E} \mathbf{B}_{\alpha} \mathrm{d} \xi ; \\
& \mathbf{K}_{\alpha d}=\frac{b \cdot h \cdot L}{2} \int_{-1}^{1} \mathbf{B}_{\alpha}^{T} \mathbf{E} \mathbf{B} \mathrm{d} \xi ; \\
& \mathbf{K}_{\alpha \alpha}=\frac{b \cdot h \cdot L}{2} \int_{-1}^{1} \mathbf{B}_{\alpha}^{T} \mathbf{E} \mathbf{B}_{\alpha} \mathrm{d} \xi .
\end{aligned}
$$

Since the elasticity matrix is symmetric $\mathbf{K}_{\mathbf{d} \alpha}=\mathbf{K}_{\alpha \mathbf{d}}$. 

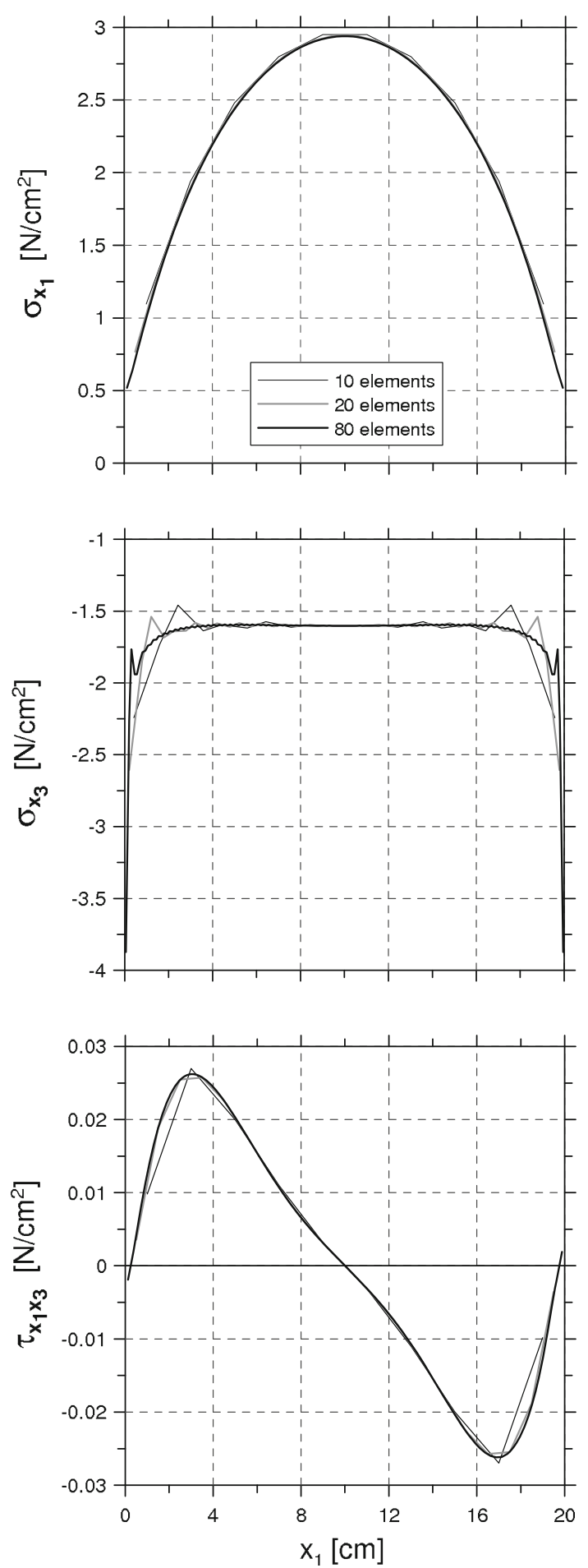

Fig. 9 Case $E_{m}=30 E_{b}$, selective reduced integration, internal stress and contact tractions

Solving Eq. 43 respect to $\boldsymbol{\alpha}$ and substituting the result in the Eqs. 42 and 43 the enlarged strain-displacement relations and the condensed form of the element equilibrium equations are obtained:
$\hat{\boldsymbol{\varepsilon}}=\left(\mathbf{B}-\mathbf{B}_{\alpha} \mathbf{K}_{\alpha \alpha}^{-1} \mathbf{K}_{\alpha d}\right) \mathbf{d}$

$\overline{\mathbf{K}} \mathbf{d}=\mathbf{f}$

with $\overline{\mathbf{K}}=\mathbf{K}_{d d}-\mathbf{K}_{d \alpha} \mathbf{K}_{\alpha \alpha}^{-1} \mathbf{K}_{\alpha d}$. The crucial point in the application of the EAS strategy is the number of additional parameters to introduce in the element formulation and the form of the strain-displacement matrix. Regarding the second point is reasonable to decompose the $\mathbf{B}_{\alpha}$ in a constant and linear part along the element, as for the matrix (32), namely

$\mathbf{B}_{\alpha}=\mathbf{B}_{\alpha 0}+\mathbf{B}_{\alpha 1} \xi$.

The satisfaction of the Taylor et al [31] conditions implies that:

$$
\begin{aligned}
\int_{-1}^{1} \mathbf{B}_{\alpha} \mathrm{d} \xi & =0 \quad \rightarrow \mathbf{B}_{\alpha 0}=\mathbf{0} \\
\boldsymbol{\alpha}^{T} \mathbf{K}_{\alpha \alpha} \boldsymbol{\alpha} & >0
\end{aligned}
$$

or that the constant part of the kinematic compatibility matrix is null and the sub-matrix $\mathbf{K}_{\alpha \alpha}$ must be positive definite.

In view of the previous result, the enlarged strain vector and the condensed stiffness matrix, after some matrix algebra manipulations, can be rewritten as follows:

$$
\begin{aligned}
& \hat{\boldsymbol{\varepsilon}}=\left[\mathbf{B}_{0}+\left(\mathbf{I}-\mathbf{C}_{\alpha}\right) \mathbf{B}_{1} \xi\right] \mathbf{d} \\
& \overline{\mathbf{K}}=b \cdot h \cdot L\left[\mathbf{B}_{0}^{T} \mathbf{E} \mathbf{B}_{0}+\frac{E}{3\left(1-v^{2}\right)} \mathbf{B}_{1}^{T}\left(\mathbf{I}-\mathbf{C}_{\alpha}\right) \mathbf{B}_{1}\right]
\end{aligned}
$$

where $\mathbf{I}$ is the $(3 \times 3)$ unit matrix and $\mathbf{C}_{\alpha}$ assumes the general form:

$\mathbf{C}_{\alpha}=\mathbf{B}_{\alpha 1}\left(\mathbf{B}_{\alpha 1}^{T} \mathbf{E} \mathbf{B}_{\alpha 1}\right)^{-1} \mathbf{B}_{\alpha 1}^{T} \mathbf{E}$

Therefore, on the base of the number of kinematical additional parameters and on shape of $\mathbf{B}_{\alpha 1}$, the matrix $\mathbf{C}_{\alpha}$ is specialized and from Eq. 50 the condensed stiffness matrix is derived. In the present study two different cases have been implemented with two and three additional kinematical parameters.

\subsubsection{Case 1: two additional kinematical parameters}

In this case the choice consists in

$\boldsymbol{\alpha}=\left[\begin{array}{ll}\alpha_{1} & \alpha_{2}\end{array}\right]^{T}, \quad \mathbf{B}_{\alpha 1}=\left[\begin{array}{ll}1 & 0 \\ 0 & 0 \\ 0 & 1\end{array}\right]$,

which from Eqs. 51 and 50 implies that

$$
\begin{aligned}
& \mathbf{C}_{\alpha}=\left[\begin{array}{lll}
1 & v & 0 \\
0 & 0 & 0 \\
0 & 0 & 1
\end{array}\right], \\
& \overline{\mathbf{K}}=b \cdot h \cdot L\left[\mathbf{B}_{0}^{T} \mathbf{E} \mathbf{B}_{0}+\frac{E}{3\left(1-v^{2}\right)} \mathbf{b}_{2}^{T} \mathbf{b}_{2}\right] .
\end{aligned}
$$




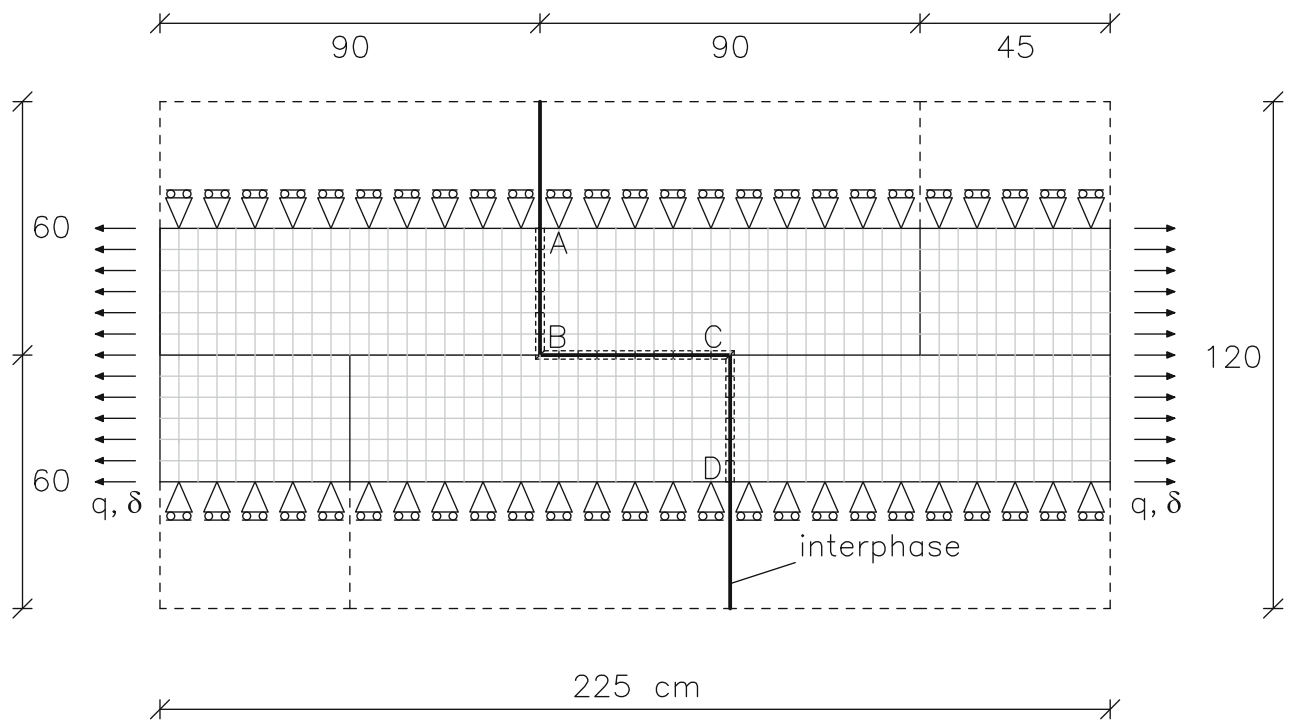

Fig. 10 Example 1: masonry specimen extracted from an infinitely long wall under tensile loading parallel to the bed joint

It is remarkable that the expression of element condensed stiffness matrix coincides with that obtained making use of the Reduced Selective Integration (RSI) technique.

\subsubsection{Case 2: three additional kinematical parameters}

The second choice consists in

$\boldsymbol{\alpha}=\left[\begin{array}{lll}\alpha_{1} & \alpha_{2} & \alpha_{3}\end{array}\right]^{T}, \quad \mathbf{B}_{\alpha 1}=\mathbf{I}$,

and the results are

$\mathbf{C}_{\alpha}=\mathbf{I}, \quad \overline{\mathbf{K}}=b \cdot h \cdot L \mathbf{B}_{0}^{T} \mathbf{E B}_{0}$.

Also in this case a result already obtained is found, in fact the condensed stiffness matrix coincides with that provided by the Reduced Integration (RI) technique.

\section{Numerical application}

The interphase finite element should be used instead of the ZTI element in all those applications where the stress state of the joint bulk material cannot be neglected.

In this section two linear elastic analyses are carried out to demonstrate the capabilities of the element when it is subjected to a more complex load combination as compression or tension and shear.

The first numerical example regards the elastic response of a masonry detail extracted from an infinitely large masonry panel made of $90 \times 60 \times 10 \mathrm{~cm}^{3}$ blocks and 0.2 and $0.3 \mathrm{~cm}$ thick bed and head joints, respectively. The masonry detail is subjected to an uniform distribution of tensile forces in the horizontal direction and the vertical deformation is not allowed.
Table 1 Example 1, elastic properties of the blocks and of the mortar

\begin{tabular}{lllll}
\hline Block & & & Mortar & \\
\cline { 1 - 2 }$E_{b}(\mathrm{MPa})$ & $v_{b}$ & & $E_{m}(\mathrm{MPa})$ & $v_{m}$ \\
\hline 5,000 & 0.2 & 1,000 & 0.2 \\
\hline
\end{tabular}

The finite element model is illustrated in Fig. 10. In the vertical direction, due to the symmetry condition, only two half units are modelled. The horizontal length of the specimen, two and a half units, is chosen according to the Saint Venant principle in order to reduce the influence of the left and right borders in the stress distribution around the potential crack zone. In $[8,9]$ this kind of simulation is used to study the tensile strength of the masonry material in the direction parallel to the bed joints.

In the previous studies, units were modelled with continuum elements and joints with interface elements. In this work the joints are simulated by means of interphase elements, therefore the response comprises the internal stress $\sigma_{x 1}$ normal to the transverse section of the joints. The elastic properties of the blocks and of the mortar joints are reported in Table 1 in terms of Young's modulus $E$ and Poisson ratio $v$.

The elastic response is illustrated in Fig. 11a. As expected, the maximum values of the normal contact traction $\sigma_{x 3}$ occur in the head joints $(\mathrm{AB}, \mathrm{CD})$ while in the same segments the contact tangential tractions $\tau_{x 1 \times 3}$ are negligible. In the bed joint normal and tangential contact tractions are of the same magnitude, the tangential one attains the maximum values at the extremal points of the segment BC. The internal stresses $\sigma_{x 1}$ are positive in the head and bed joints and the values are 

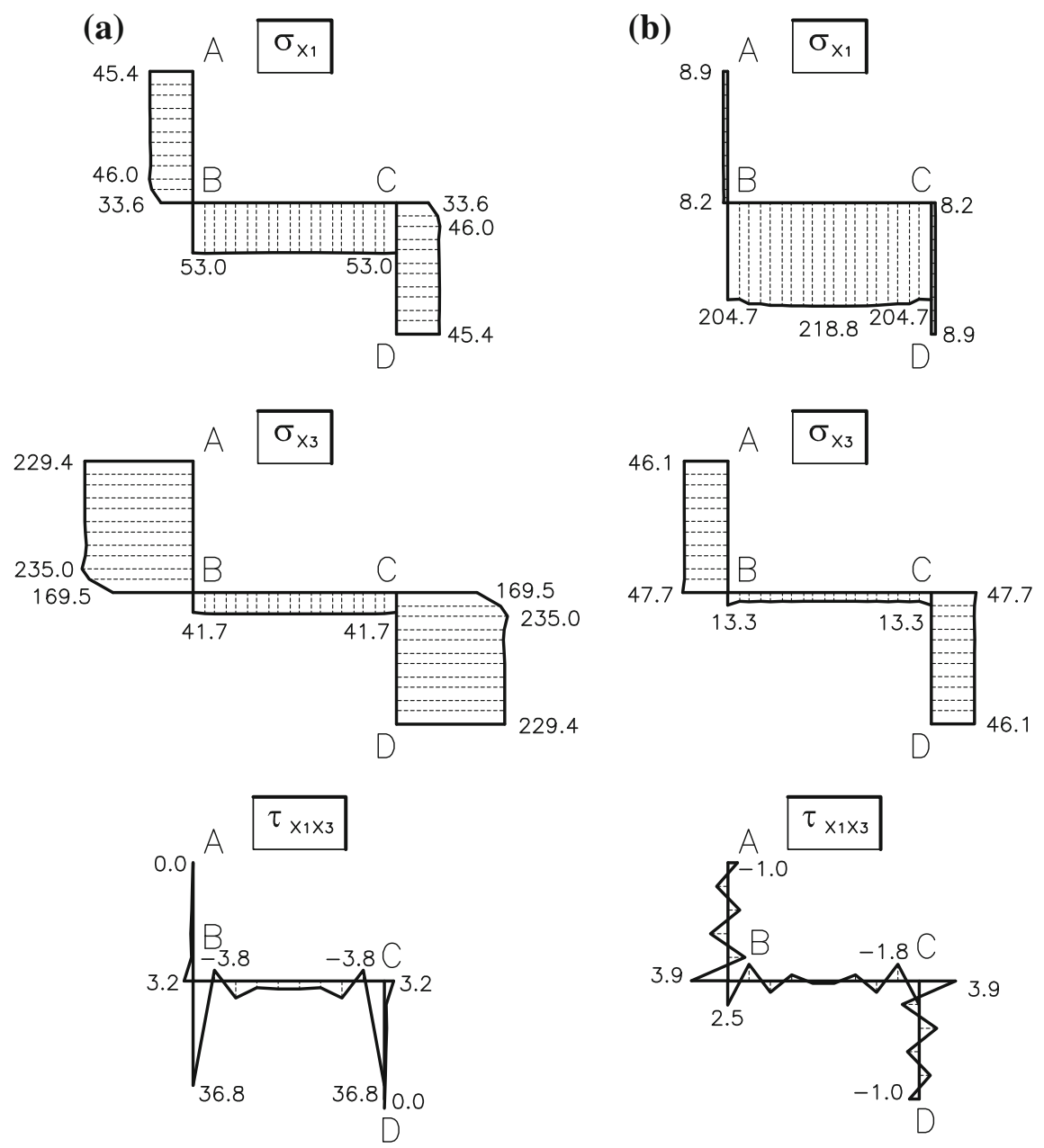

Fig. 11 Example 1: internal stress $\sigma_{x 1}$, normal $\sigma_{x 3}$ and tangential $\tau_{x 1 x 3}$ tractions $\left(\mathrm{N} / \mathrm{cm}^{2}\right),(\mathbf{a}) E_{b}>E_{m},(\mathbf{b}) E_{m}>E_{b}$

similar. This because of presence of the constraints applied to the horizontal boundaries.

The results of an interesting numerical experiment is illustrated in Fig. 11b representing the stress response of the same finite element model after exchanging the elastic modulus of the block with the elastic modulus of the mortar, and vice versa. Now the internal stress $\sigma_{x 1}$ in the bed joint assumes a considerable value together with a drastic reduction of the normal contact traction $\sigma_{x 3}$ in the head joints. The contact tangential tractions are negligible along the joints.

The latter example regards the mechanical response of the masonry panel illustrated in Fig. 12. The specimen consists of a pier built up with 18 courses of clay bricks $(20.4 \times$ $\left.9.8 \times 5.0 \mathrm{~cm}^{3}\right)$ and $1 \mathrm{~cm}$ thick mortar joints. The geometry of the wall and the boundary conditions imposed are shown in Fig. 12. The wall is pre-stressed by applying an uniform pressure $q$ at the top of the element and a concentrated shear force $F$ is located at the top course. At the top horizontal side of the wall the vertical degrees of freedom are constrained.
The finite element analysis was performed modelling the single brick with $8 \times 4$ plane stress continuum elements and the bed and head joints with 8 and 4 interphase elements, respectively. The elastic properties of the blocks and of the mortar joints are reported in Table 2.

The results, also in this example, are expressed in terms of contact tractions and internal stress and the diagrams along three bed joints (A-A, B-B and C-C) are depicted in Fig. 13 . Due to the bending moments acting at the top and at the bottom of the panel, the cross sections $\mathrm{A}-\mathrm{A}$ and $\mathrm{C}-\mathrm{C}$ are divided in the compression and tension part. In the bed joint located in the middle of the panel (B-B) the bending moment is zero and the panel is almost uniformly compressed. The normal internal stress shows a similar profile to the normal contact stress and the same sign, since the block material is stiffer than the mortar. It must be noticed that in both diagrams sort of jumps are present where the head joints intersect the bed joint. Finally, the contact tangential stress in the bed joint B-B assumes a parabolic-like profile similar to that obtained by using the Jourawsky's shear theory. This feature is less 


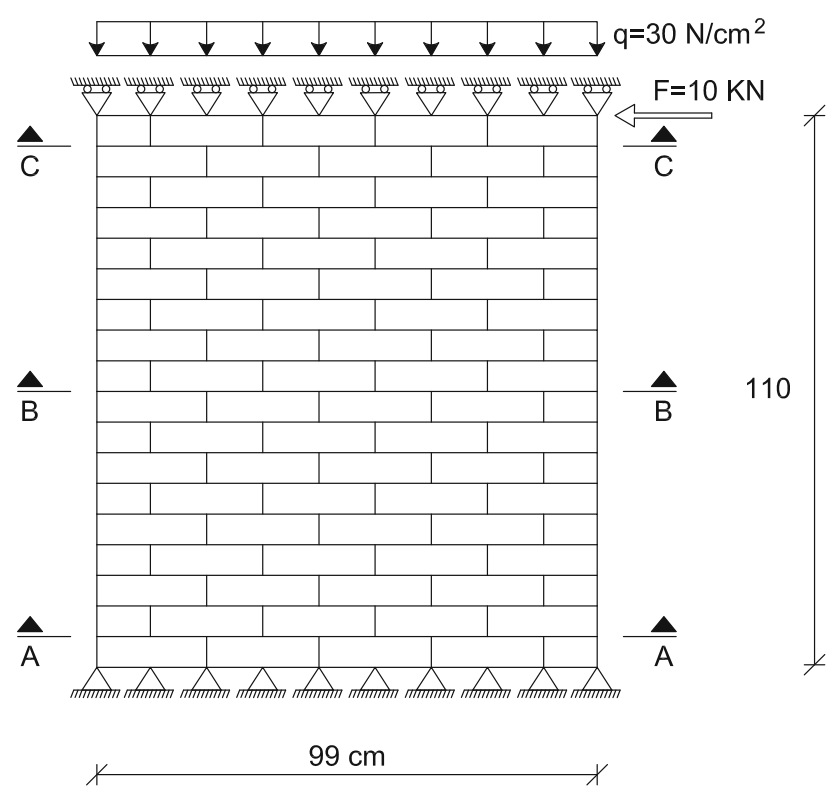

Fig. 12 Example 2: masonry wall, geometry, texture, boundary and loading conditions

Table 2 Example 2, elastic properties of the blocks and of the mortar

\begin{tabular}{|c|c|c|c|}
\hline \multicolumn{2}{|l|}{ Block } & \multicolumn{2}{|l|}{ Mortar } \\
\hline$E_{b}(\mathrm{MPa})$ & $v_{b}$ & $E_{m}(\mathrm{MPa})$ & $v_{m}$ \\
\hline 16,700 & 0.15 & 820 & 0.14 \\
\hline
\end{tabular}

pronounced in the top and bottom bed joint due to the proximity to the boundary of the model.

\section{Conclusions}

The interphase model represents the enhancement of the zero-thickness interface model and is particularly useful for those cases where the joint internal stresses and strains play a crucial role in the response of the heterogeneous materials. One of these cases is the uniaxial compression test of masonry material where the mechanical elastic response and the failure mode of the specimen strongly depend on the internal stress state within the mortar layer.

For the purpose of numerical mesomodelling of structures made up of composite materials, in the present work the interphase element is proposed which can be easily derived from the classical interface element opportunely modifying the strain-displacement relations.

The numerical performance of the element is investigated in detail making use of a simple elastic patch test. Adopting the standard Gauss quadrature for the stiffness matrix integration, the element shows unacceptable oscillation of the tangential contact stress profile which has also been observed
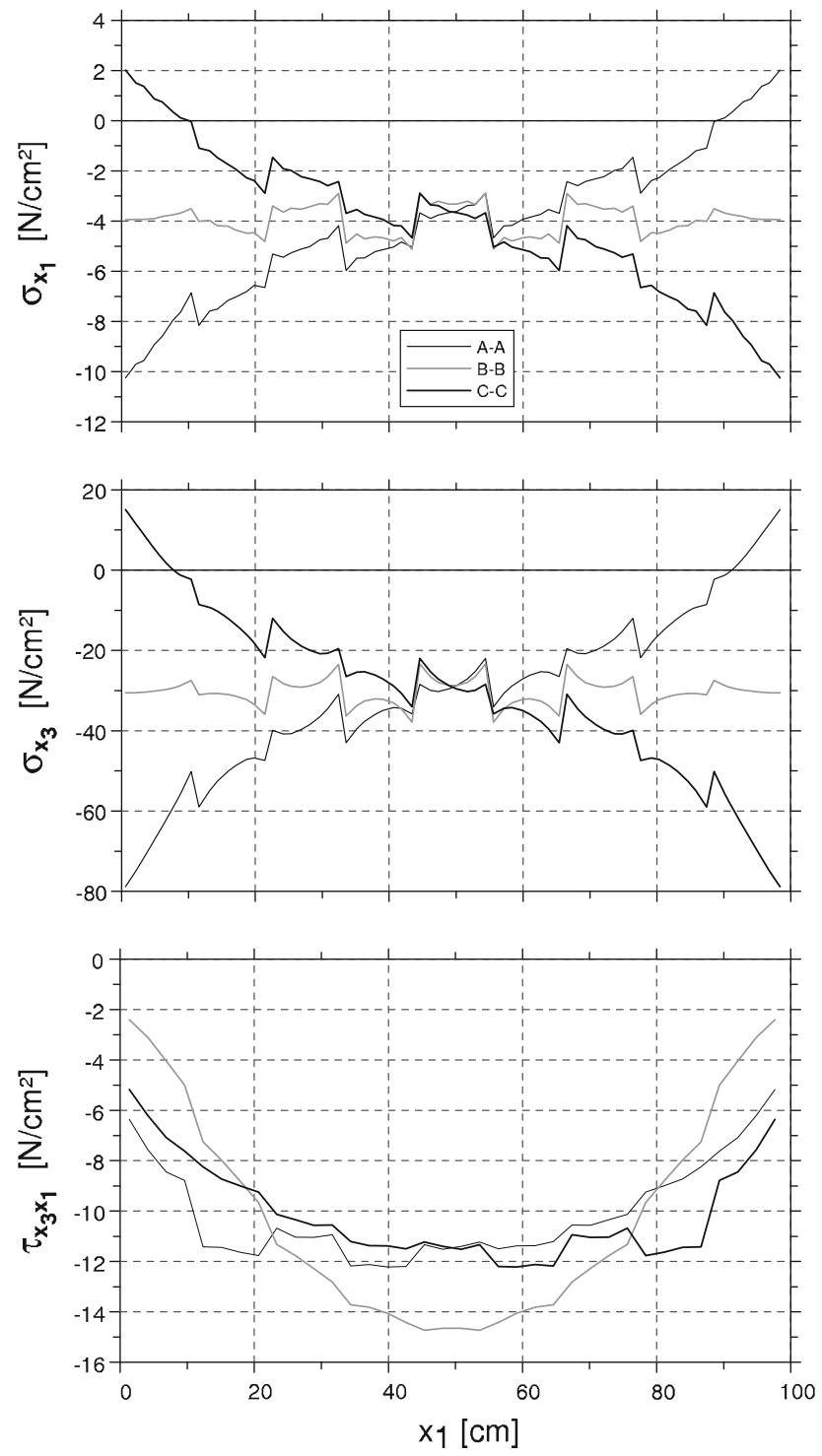

Fig. 13 Example 2: internal stress $\sigma_{x 1}$, normal $\sigma_{x 3}$ and tangential $\tau_{x 1 x 3}$ tractions in the bed joints $\mathrm{A}-\mathrm{A}, \mathrm{B}-\mathrm{B}$ and $\mathrm{C}-\mathrm{C}$

for the interface element and for the $2 \mathrm{D}$ and $3 \mathrm{D}$ continuum thin elements.

The numerical defect is justified by the shear locking of the element and the classical numerical strategies to avoid the phenomenon are applied with success. In particular, the Selective Reduced Integration technique (RSI) and the EAS with two additional kinematical parameters are found effective. Both techniques lead to the same result in terms of stiffness matrix expression but the RSI approach is preferred because no additional variables are required and the stiffness matrix has not to be modified, important point for non-linear applications.

Finally, two elastic numerical applications to the case of structure made of masonry material are presented. The 
numerical results attest the effectiveness of the element also in presence of complex load combinations.

Without any doubt the interphase element opens new scenarios in the mechanics of joints constituted by a thin layer of a third material. The distinction of contact tractions and internal stresses allows to introduce different failure conditions for the physical interfaces and for the bulk material and the damage of the material can be described separately from the loss of adhesion in correspondence of the joint-adherent contact surface. The joint stiffness degradation can be related to the damage of the joint material and the attainment of the yield condition at the physical interface can be responsible of the onset of irreversible displacement discontinuities.

Acknowledgments The authors acknowledge the financial support given by the Italian Ministry of Education, University and Research (MIUR) under the PRIN07 project 2007YZ3B24, "Multiscale problems with complex interactions in Structural Engineering".

\section{References}

1. Del Piero G (1989) Constitutive equation and compatibility of external loads for linear elastic masonry-like materials. Meccanica 24:150-162

2. Pietruszczak S, Niu X (1992) A mathematical description of macroscopic behaviour of brick masonry. Int J Solids Struct 29: 531-546

3. De Buhan P, De Felice G (1997) A homogenization approach to the ultimate strength of brick masonry. J Mech Phys Solids 45:1085-1104

4. Bolzon G, Ghilotti D, Maier G (2002) Strength of periodic elasticbrittle composites evaluated through homogenization and parameter identification. Eur J Mech A 21:355-378

5. Page AW (1978) Finite element model for masonry. J Struct Eng (ASCE) 104(8):1267-1285

6. Stankowski T, Runesson K, Sture S (1993) Fracture and slip of interfaces in cementitious composites. J Eng Mech (ASCE) 119(2):292-314

7. Lofti HR, Shing PB (1994) Interface model applied to fracture of masonry structures. J Struct Eng (ASCE) 120(1):63-80

8. Lourenco PB, Rots JG (1997) Multisurface interface model for analysis of masonry structures. J Eng Mech (ASCE) 123(7):660668

9. Giambanco G, Rizzo S, Spallino R (2001) Numerical analysis of masonry structures via interface models. Comput Methods Appl Mech Eng 190:6493-6511

10. Allix O, Ladeveze P (1992) Interlaminar interface modelling for the prediction of laminates delamination. Compos Struct 22: 235-242

11. Allix O, Ladeveze P, Corigliano A (1995) Damage analysis of interlaminar fracture specimens. Compos Struct 31:61-74

12. Allix O, Leveque D, Perret L (1998) Identification and forecast of delamination in composite laminates by an interlaminar interface model. Compos Sci Technol 58:671-678
13. Corigliano A, Allix O (2000) Some aspects of interlaminar degradation in composites. Comput Methods Appl Mech Eng 2:203-224

14. Alfano G, Crisfield MA (2001) Finite element interface models for the delamination analysis of laminated composites: mechanical and computational issues. Int J Numer Methods Eng 50:1701-1736

15. Massabo' R, Mumm D, Cox B (1998) Characterising mode II delamination cracks in stitched composites. Int J Fract 92(1):1-38

16. Ming H, Cox BN (1998) Crack bridging by through-thickness reinforcement in delaminating curved structures. Composites A 29:377-393

17. Cottone A, Turetta T, Giambanco G (2007) Delamination study of through-thickness reinforced composite laminates via two-phase interface model. Composites A 38:1985-1995

18. Herrmann LR (1978) Finite element analysis of contact problems. ASCE J Eng Mech 104:1043-1057

19. Zavarise G, Wriggers P, Schrefler BA (1998) A method for solving contact problems. Int J Numer Methods Eng 42:473-498

20. Griffiths DV (1985) Numerical modelling of interfaces using conventional finite elements. In: Proceedings of 5th international conference on Numerical Methods in Geomechanics, Nagoya, pp 837-844

21. Goodman RE, Taylor RL, Brekke TL (1968) A model for the mechanics of jointed rock. ASCE J Soil Mech Found Div 94: 637-659

22. Beer G (1985) An isoparametric joint/interface element for finite element analysis. Int J Numer Method Eng 21:585-600

23. Ortiz M, Pandolfi A (1999) Finite-deformation irreversible cohesive elements for three-dimensional crack propagation analysis. Int J Numer Methods Eng 44:1267-1282

24. Schellekens JCJ, de Borst R (1993) On the numerical integration of interface elements. Int J Numer Methods Eng 36:43-66

25. Kaliakin VN, Li J (1995) Inside into deficiencies associated with commonly used zero-thickness interface elements. Comput Geomech. 17:225-252

26. Giambanco G, Mroz Z (2001) The interphase model for the analysis of joints in rock masses and masonry structures. Meccanica 36:111-130

27. Zienkiewicz OC, Taylor RL, Too JM (1971) Reduced integration technique in general analysis of plates and shells. Int J Numer Methods Eng 3:275-290

28. Hughes TJR, Cohen M, Harouna M (1978) Reduced and selective integration techniques in the finite element analysis of plates. Nucl Eng Des 46:203-222

29. Simo JC, Rifai MS (1990) A class of mixed assumed strain methods and the method of incompatible modes. Int J Numer Methods Eng 29:1595-1638

30. Arnold DN, Brezzi F (1997) The partial selective reduced integration method and applications to shell problems. Comput Struct 64:879-880

31. Taylor RL, Beresford PJ, Wilson EL (1976) A non-conforming element for stress analysis. Int J Numer Methods Eng 10: 1211-1219

32. Lourenco PB, Rots JG, Vander Pluijm R (1999) Understanding the tensile behavior of masonry panel parallel to the bed joints: a numerical approach. Mason Int 12:96-103 